\title{
Almohadilla Desechable Biodegradable a ser usada en un Pañal Ecológico
}

\author{
Cuauhtémoc Pozos Vázquez, PhD, \\ Patricia Blanco Padilla, PhD, \\ Martha Ofelia Mercado González, \\ Gustavo Abraham Vélez Martínez,
} Instituto de Estudios Superiores de Tamaulipas (IEST), Altamira, Tamaulipas, México

Doi: 10.19044/esj.2018.v14n12p37 URL:http://dx.doi.org/10.19044/esj.2018.v14n12p37

\begin{abstract}
The disposition of urban solid waste is a major problem around the world today due to the large amount of spaces it occupies and the negative effects it has on the environment. These effects include pollution, most especially when it comes in contact with terrestrial and aquatic ecosystems. One of the solid wastes that are generated in large quantities is the baby diapers. This, however, is because of its high demand for use. Plastics with which conventional disposable diapers are made can take many years to degrade. An attractive alternative to reduce its contaminating effect involves the use of reusable diapers in which the absorbent(insert) is not discarded. Nevertheless, one of the major disadvantages is that it requires a lot of washing after its use. Thus, it is time-consuming as the diapers have to be washed with water and detergents. This paper focuses on an attractive alternative of a new $100 \%$ biodegradable absorbent which was produced. This alternative can be disposed without having any significant impact on the environment. Through this way, there is a promotion of the idea of using an ecological diaper with a disposable absorbent (insert) made from natural and biodegradable materials with which it would avoid exposing babies to the chemical components of conventional disposable diapers. In addition, costs are minimized since the diaper is not completely discarded; thus, only the absorbent is removed.
\end{abstract}

Keywords: Water hyacinth, biosorption, biomaterials

\section{Resumen}

La disposición de los desechos sólidos urbanos es una problemática mundial que se intenta resolver mediante diferentes alternativas debido a los grandes espacios ocupados para su confinamiento y sus efectos negativos al 
medio ambiente, tales como la contaminación, al entrar en contacto con ecosistemas terrestres y acuáticos. Uno de los desechos sólidos que se generan en grandes cantidades son los pañales de bebés debido a la alta demanda de su consumo. Los plásticos con los cuales se elaboran los pañales desechables convencionales pueden tomar muchos años en degradarse. Una alternativa atractiva para reducir su efecto contaminante, es el uso de pañales reutilizables en los cuales el absorbente (inserto) no se desecha pero requiere una etapa de lavado posterior a su utilización con lo cual consumiría agua y detergentes. En el presente estudio se elaboró un nuevo absorbente $100 \%$ biodegradable, el cual puede ser desechado sin afectar al medio ambiente. De esta manera se promueve la idea de usar un pañal ecológico con un absorbente (inserto) desechable elaborado a partir de materiales biodegradables y naturales con el cual se evitaría exponer a los bebés a los componentes químicos presentes en los pañales desechables convencionales. Adicionalmente se minimizan los costos ya que no se desecha el pañal por completo, solo se elimina el absorbente.

Palabras-claves: Lirio acuático, biosorción, biomateriales

\section{Introducción}

Hoy en día, el conocimiento sobre los objetos que usamos que contaminan más el medio ambiente es reducido, y resulta realmente asombroso cuando nos enfrentamos a ello. Tal es el caso del coste ecológico de los pañales. Los pañales desechables convencionales se producen usando como materia prima la celulosa, lo que supone que para hacer los pañales que usará un niño en sus primeros años de vida se requerirá de la tala de árboles.

Por otra parte, los pañales reutilizables usan un $90 \%$ menos de materia prima en su producción y también menos energía al fabricarse. Además estos pañales crearán un $60 \%$ menos de basura ya que al coste ecológico del pañal desechable durante su producción hay que añadir que los mismos suponen varias toneladas de desechos que van a la basura por cada niño y que se requieren muchos años para su degradación (Rojas González \& Osswald, 2015). En el caso de los pañales reutilizables, si bien es cierto que deben ser lavados, su limpieza no supone un gran coste ecológico.

En la fabricación de los pañales desechables generalmente se utilizan componentes químicos entre los que se destacan las dioxinas, el tributil-estaño (el cual es un contaminante tóxico), además de otros materiales incluidos los plásticos. Estos materiales difícilmente se descomponen completamente, por lo cual contaminan los suelos y aguas, esto de acuerdo con la agencia de Protección Medioambiental (EPA, por sus siglas en inglés).

Asimismo, los pañales desechables han sido tratados con productos derivados del cloro, blanqueantes, que actúan sobre la pasta de papel de la 
celulosa y la modifican. Esto produce vertidos de productos tóxicos que llegan al medio ambiente y que permanecen en contacto con la piel del niño (Smith, 2013). De igual manera se usa como absorbente un polímero sintético, el poliacrilato de sodio, que es el producto que gelifica la orina del bebé y puede llegar a resecar la piel. Los costes ecológicos del uso de pañales son considerables, y mucho mayores en el caso de los pañales desechables convencionales.

Según el Programa de Naciones Unidas para el Medio Ambiente (PNUMA) y el gobierno de Suecia, el 14\% de los residuos sólidos urbanos generados en México corresponden a pañales desechables, lo cual constituye un reto en materia de manejo de residuos en los rellenos sanitarios. Los que no llegan a los vertederos son tirados en ríos, barrancas, al aire libre, coladeras y al mar ocasionando graves daños al ecosistema. En el país anualmente se consumen dos millones 160 mil pañales desechables y un bebé mexicano utiliza en promedio en tres años, seis mil 700 pañales. En base a estos consumos per cápita México ocupa el cuarto lugar a nivel mundial de pañales vendidos según un reporte realizado por Euromonitor en el 2013 como se muestra en la Gráfica 1.

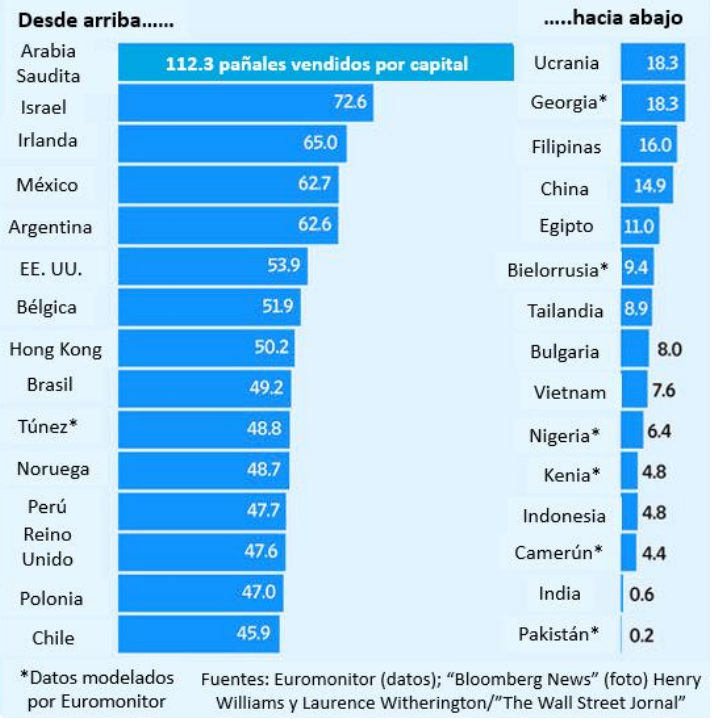

Gráfica 1. Datos de consumo de pañales per cápita por países.

Por otra parte, varios son los ejemplos del uso del tallo maduro del lirio acuático como material lignocelulósico sorbente de sustancias líquidas. Tal es el caso del control de derrames de combustibles (gasolina, diésel y turbosina), en donde se encontró que dicho tallo sorbió de 5 a 6 veces su peso de combustible por gramo de sorbente (Márquez Rivera \& Arguelles Robles, 2017). Asimismo, este mismo biomaterial es capaz de eliminar un colorante 
modelo como el azul de metileno de soluciones acuosas obteniéndose una capacidad máxima de biosorción de $159 \mathrm{mg} / \mathrm{g}$ (Pozos Vázquez \& Bollnow Cortes, 2017).

El presente proyecto propone como idea innovadora el uso de biomateriales (Lirio acuático-Eichhornia crassipes-y carboximetilcelulosa, $C M C$ ) como relleno absorbente para su utilización en almohadillas desechables y biodegradables en pañales ecológicos con la finalidad de contribuir a la reducción de la contaminación del medio ambiente (Figura 2).

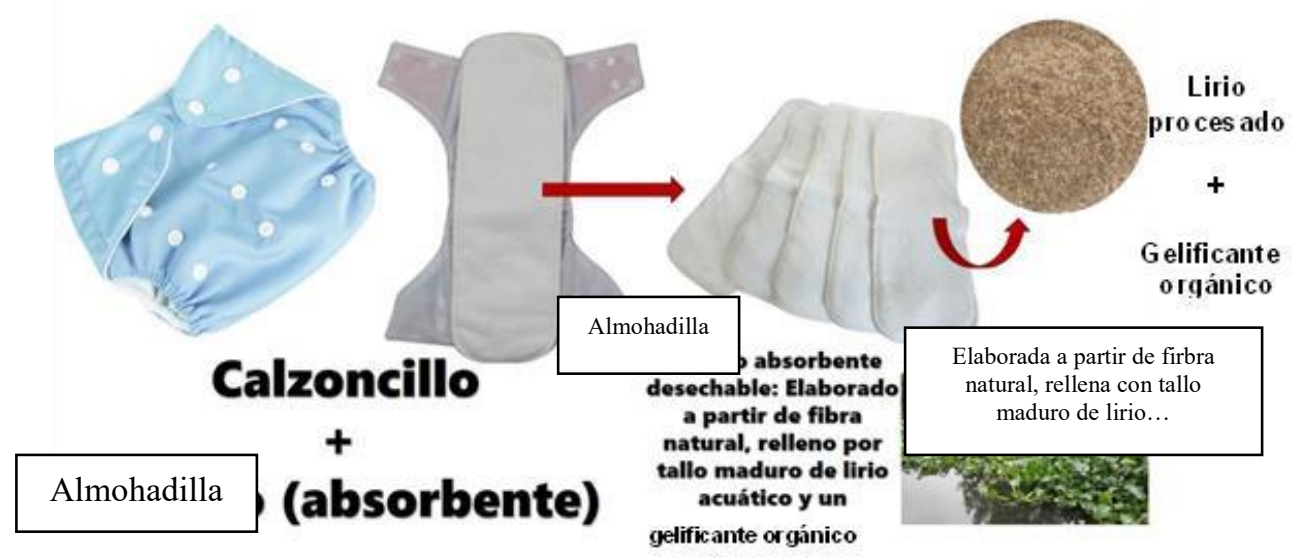

Figura 2. Propuesta de una nueva almohadilla para ser aplicada en un pañal ecológico.

\section{Objetivo general}

Elaborar una almohadilla absorbente biodegradable para pañales.

\section{Objetivos específicos}

1. Determina la capacidad máxima de absorción de orina que posee el tallo maduro del Lirio Acuático.

2. Identificar mediante una encuesta el impacto ambiental de la propuesta con respecto a los pañales convencionales.

\section{Parte experimental}

Las muestras de tallos maduros (alrededor de $60-70 \mathrm{~cm}$ de longitud) del Lirio Acuático - recolectados de la laguna del Champayan, Ciudad Tampico, Tamaulipas- fueron seleccionadas previamente a las experiencias de absorción ,sin indicios de descomposición o putrefacción, y fueron lavadas, cortadas, secadas a $60^{\circ} \mathrm{C}$ durante una noche, y luego molidas y cernidas en un tamiz con la finalidad de homogenizar las partículas a un tamaño promedio de $0.5 \mathrm{~mm}$ (granulometría seleccionada). Se requirió una etapa de extracciones líquido-sólido (método Soxhlet) en el biomaterial tamizado con agua desmineralizada en una primera etapa y subsecuentemente con etanol y hexano (Figura 3), con la finalidad de remover los componentes de la planta 
que impartan coloración al momento de realizar las experiencias de absorción y que pudieran afectar el desempeño del biomaterial como absorbente en un pañal ecológico.

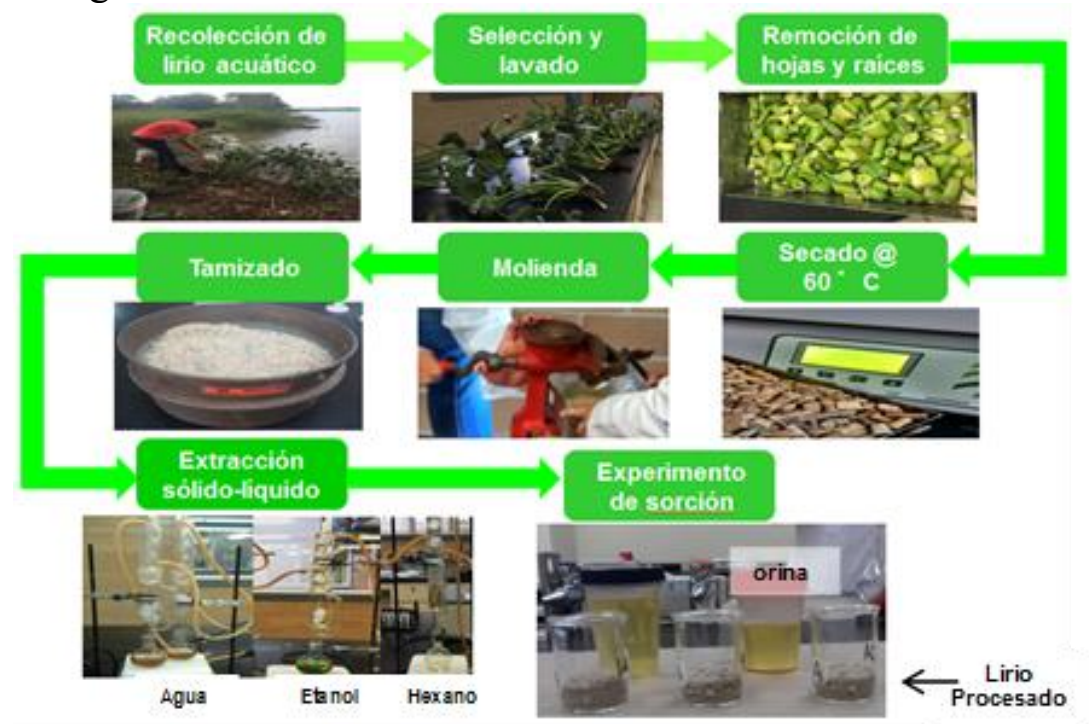

Figura 3. Diagrama de flujo del desarrollo experimental.

\section{Pruebas de absorción}

La capacidad máxima de sorción $\left(\mathrm{C}_{\mathrm{t}}\right)$ fue evaluada adaptando la norma ASTM F-726 y el protocolo canadiense "Oil Spill Sorbents: Testing Protocol and Certification Listing Program" (Leiva-Mas, Martínez Nodal, EsperanzaPérez, Rodríguez-Rico, \& Gordiz-García, 2012) en el cual se determina la masa de orina sorbida por gramo de material sorbente mediante la ecuación 1.

$$
\text { (1) } \quad C_{t}=\frac{m_{t}-m_{0}}{m_{0}}
$$

\section{Donde:}

$\mathrm{m}_{\mathrm{t}}=$ Peso del material impregnado (peso del sorbente y orina sorbida). $\mathrm{m}_{0}=$ Peso del material sorbente seco.

El procedimiento para la prueba de la capacidad máxima de sorción sin agitación previa (en reposo) fue el siguiente: La orina se dejó en contacto durante 24 horas con $1 \mathrm{~g}$ del tallo maduro del lirio acuático en un vaso de precipitado a presión atmosférica y temperatura ambiente, para luego ser decantado hasta no observar escurrimiento de la orina (orina removida). Se cuantificó la ganancia en peso por el método gravimétrico; es decir, el peso de la muestra con la orina sorbida (material impregnado). Se realizaron las pruebas por triplicado (Figura 4). Se utilizó una balanza analítica Marca Velab Modelo Ve-210 y para el análisis estadístico de los datos de la encuesta se 
utilizó Microsoft Excel. Se observaron aspectos estructurales del biomaterial (Lirio) mediante microscopía electrónica de barrido (SEM). Para ello se utilizó el microscopio marca Velab VE-653.
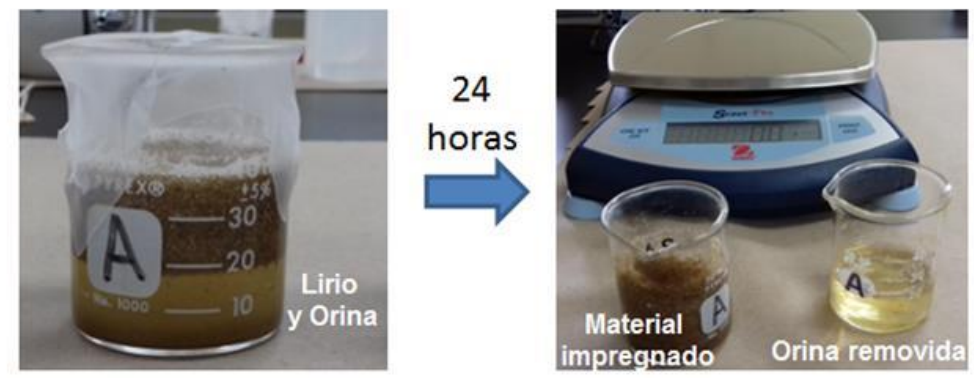

Figura 4. Prueba de absorción en batch sin agitación.

\section{Resultados y discusión}

La capacidad máxima de absorción de orina con tallo maduro de Lirio Acuático fue de $16.5 \pm 1.1 \mathrm{~g}$ de orina/ $\mathrm{g}$ de absorbente. El tejido vegetal del tallo maduro del Lirio Acuático está constituido por aerénquima, es decir, cámaras de aire, lo que le permite a la planta flotar sobre las lagunas y ríos, cámaras que se muestran en la fotografía de la Figura $5 \mathrm{a}$ al haber realizado un corte transversal del tallo. Por tal motivo, las mismas sirven como reservorios de la orina lo que le otorga esa notable capacidad de impregnación al entrar en contacto con ella. De igual manera, a nivel microscópico mediante microscopía electrónica de barrido se observan dichas cavidades con un objetivo de 100x y un poder de resolución de $4 \AA \AA$ en el tallo maduro procesado mediante el empleo del azul de metileno en fase acuosa usado como tinte de contraste, lo cual facilitó la observación (Figura $5 b$ ).
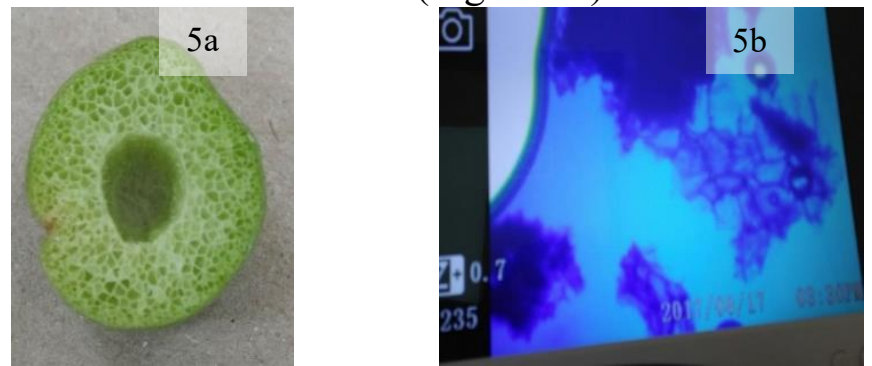

Figura 5. Fotografías del tallo maduro del lirio acuático a) corte transversal y b) microscopía electrónica de barrido del tallo procesado.

Por tal motivo, con el fin de incrementar la capacidad de absorción y favorecer la gelificación de la orina para minimizar escurrimientos en el pañal , se formuló el lirio con carboximetilcelulosa, CMC (Ramos, García, Sandoval, Arellano, \& Antonio, 2014), un polisacárido que posee la capacidad de gelificar al entrar en contacto con líquidos a temperatura ambiente (sin 
necesidad de calentar). En la Figura 6 se muestra la estructura química del gelificante orgánico utilizado, es decir, la carboximetilcelulosa, CMC.

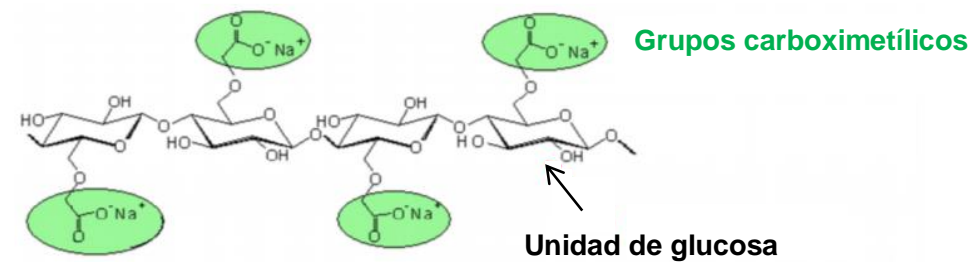

Figura 6. Esquema de la estructura química del polisacárido carboximetilcelulosa.

Se realizó una nueva experiencia de absorción pero ahora con $0.9 \mathrm{~g}$ de Lirio y $0.4 \mathrm{~g}$ de CMC lográndose en esta ocasión absorber $26 \mathrm{~g}$ de orina. Un pañal desechable del mercado en promedio absorbe alrededor de $36 \mathrm{~g}$ de orina.De esta manera, incrementando la cantidad de lirio se podría igualar esa cantidad; es decir, formular un absorbente con $1.5 \mathrm{~g}$ de lirio y $0.4 \mathrm{~g}$ de CMC. Para esta última formulación se obtuvo una sorción de $35.7 \pm 8.6 \mathrm{~g}$ de orina. La amplia dispersión para este valor promedio (en comparación con la prueba de absorción del lirio sin CMC) es debida a la notable dificultad de remover la orina gelificada mediante decantación, antes de pesar el material impregnado (Figura 7). Este resultado es interesante para el desempeño de la almohadilla en el pañal, ya que ayudaría a minimizar los escurrimientos debido a la alta gelificación de la orina.

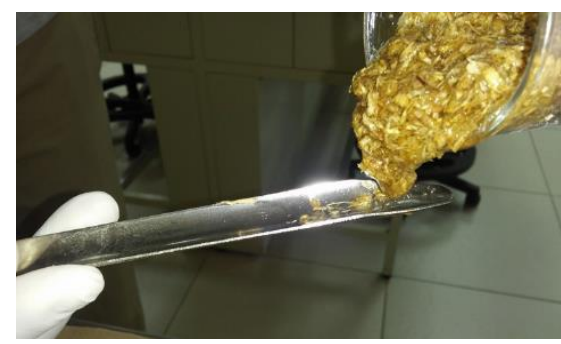

Figura 7. Formulación gelificada a partir de $1.5 \mathrm{~g}$ de lirio y $0.4 \mathrm{~g}$ de CMC.

Basándose en un pañal desechable convencional de recién nacido, el cual en promedio posee $10 \mathrm{~g}$ de absorbente y $1.7 \mathrm{~g}$ de gelificante sintético, se propone introducir $15 \mathrm{~g}$ de tallo maduro de Lirio acuático y $4 \mathrm{~g}$ de $\boldsymbol{C M C}$ previamente mezclados en el interior de una funda a base de tela de arroz, algodón o bambú y de esa manera confeccionar la almohadilla absorbente desechable . De esta manera se podría evitar la práctica de dejar al bebé en contacto con un pañal desechable convencional superabsorbente durante tiempos prolongados y contribuir así a reducir el riego de que el bebé contraiga una infección del tracto urinario (Daulay, y otros, 2013), ya que se utilizaría una nueva almohadilla biodegradable cada vez que orine el bebé. 


\section{Investigación de campo}

Para conocer si el nuevo producto desarrollado (almohadilla absorbente desechable y biodegradable) podría tener un impacto social se realizó una encuesta de tipo no probabilístico por disponibilidad a 98 individuos (sólo padres de bebés o niños de hasta 4 años de edad) en la zona conurbada de Tampico, Madero y Altamira del estado de Tamaulipas.

La encuesta es la siguiente:

\section{Encuesta estadistica sobre pañales desechables}

Objetivo: Recolectar información sobre los hábitos de compra de pañales en la zona conurbada de Tampico, Madero y Altamira

1. Edad ( ) Menor de 18 años ( )Entre 18 y 25 años ( )Entre 26 y 30 años ( ) Entre 31 y 35 años ( )Entre 36 y 40 años （） Mayor de 40 años

2. Sexo ( ) Masculino ( )Femenino

3. Estado civil （ )Soltero （）Casado （）Divorciado （）Unión libre ( )Viudo

4. Ocupación （）Estudiante （）Profesionista （）Desempleado ( )Ama de casa ( )Empresario ( )Empleado

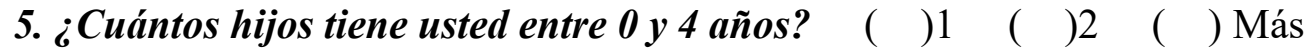
de 3

6. ¿Qué edad tiene su hijo? ( )Recién nacido ( )Menos de un año ( ) Más de 1 y menos de 2 años ( )Más de 2 y menos de 3 años

( )Más de 3 años

7. ¿Con qué frecuencia realiza actividades fuera de casa con sus hijos de 0 a 4 años (viajar, ir al supermercado, ir a espacios recreativos, etc.)?

( )1 vez por semana ( )2 veces por semana ( )3 veces por semana ( )Más de 3 veces por semana ( )Ninguna vez

8. ¿Qué tipo de pañal utiliza su bebé o niño? （）Desechable （）No desechable (de tela) ( )Biodegradable (ecológicos) ( )Otro

9. ¿Qué marca de pañales usualmente compra?

10. ¿Dónde compra los pañales? （ )Tiendas de supermercado

( )Farmacias ( )Tiendas de conveniencia ( )Tienda en línea (internet)

( )Los elabora usted mismo (tela u otro material)

11. ¿Cuántos pañales en promedio utiliza durante la semana por bebé o niño? ( )Más de 40 ( )de 30 a $40 \quad$ ( ) de 20 a $30 \quad$ ( ) de 10 a 20 ( ) de 1 a 10

12. ¿Con qué frecuencia compra los pañales? （）Diario （）1 vez por semana ( )Cada 2 semanas ( )Cada mes ( )Otro, ¿cada cuánto?

13. ¿Aproximadamente cuánto dinero en pesos gasta en pañales semanalmente?

( )Más de $\$ 500 \quad$ ( )De $\$ 500$ a $\$ 400 \quad$ ( )De $\$ 400$ a $\$ 300$ 
( )De $\$ 300$ a $\$ 100 \quad(\quad)$ Menos de $\$ 100$

14. ¿Conoce los pañales biodegradables (ecológicos)? （）Sí （）No

15. ¿Es importante para usted que los pañales sean biodegradables?

( )Sí ( )No ( )Me es indiferente

16. ¿Alguna vez ha comprado pañales biodegradables (ecológicos)?

( )Sí ( )No

17. Si el precio no es un factor de decisión de compra, ¿Le interesaría comprar un pañal biodegradable? （ )Muy de acuerdo （）De acuerdo ( ) Ni de acuerdo ni en desacuerdo ( )En desacuerdo ( )Muy en desacuerdo

18. ¿Ha presentado alguna vez su hijo(a) un malestar debido al uso de pañales desechables convencionales? ( )Sí, ¿cuáles?

( ) No

19. Los pañales desechables convencionales se fabrican con productos sintéticos, ¿le interesaría comprar pañales biodegradables elaborados a partir de materiales naturales?

( )Sí ( )No, ¿Por qué?

20. ¿Le agrada saber que el uso de pañales biodegradables (ecológicos) contribuye a reducir la contaminación del medio ambiente? ( )Muy de acuerdo ( )De acuerdo ( )Ni de acuerdo, ni en desacuerdo

( )En desacuerdo ( )Muy en desacuerdo

21. ¿Le gustaría economizar al reducir el gasto en la compra de pañales?

( )Muy de acuerdo ( )De acuerdo ( )Ni de acuerdo, ni en desacuerdo ( )En desacuerdo ( )Muy en desacuerdo

22. ¿Estaría dispuesto(a) a usar un pañal, el cual consiste de un calzoncillo reutilizable y una almohadilla retirable después del uso. （ )Sí

( )No, ¿Por qué?

23. Estaría dispuesto a lavar el calzoncillo reutilizable. ( )Sí ( )No, ¿Por qué?

24. ¿Qué características prefiere en un pañal?

25. ¿Qué medio utiliza para obtener información de pañales?

( )Internet ( )Televisión ( )Revistas/Periódicos ( )Volantes

( ) Otros

Al realizar el tratamiento estadístico de los datos de la pregunta 19 (Gráfica 8) se pudo percatar que el nuevo producto tendría aceptación en el mercado en base a la muestra representativa a la que se aplicó la encuesta (92\% de los encuestados). 


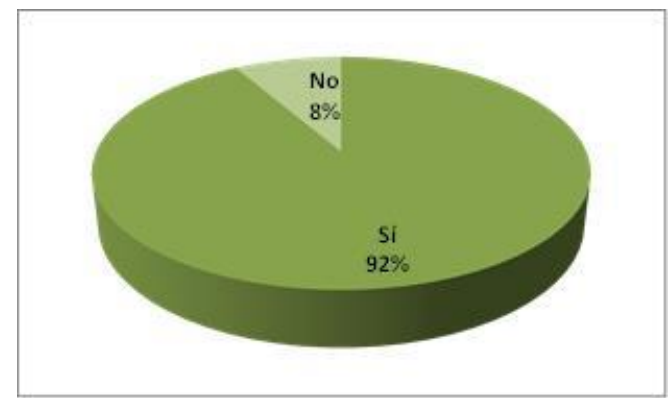

Gráfica 8. Gráfica de sectores del porcentaje de aceptación de pañales elaborados con materiales naturales (pregunta 19 de la encuesta).

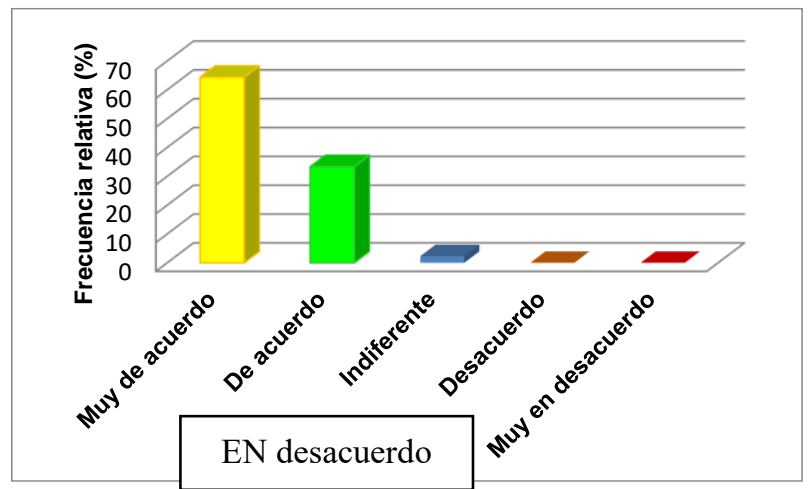

Gráfica 9. Gráfica de sectores con respecto al interés de las personas por consumir pañales que no contaminarían al medio ambiente (pregunta 20 de la encuesta).

Asimismo, el $64 \%$ de los encuestados está muy de acuerdo con el hecho de que el uso de pañales biodegradables contribuye a reducir la contaminación y el 33\% está sólo de acuerdo, lo cual engloba un $97 \%$ de los encuestados (Gráfica 9). Este resultado da una idea de la percepción y consciencia que tienen los padres de los bebés en proteger y contribuir al cuidado del medio ambiente mediante el uso de un pañal ecológico. Por último, aproximadamente el $70 \%$ de los encuestados gastan entre $\$ 100$ y $\$ 400$ pesos por semana en pañales convencionales (pregunta 13 de la encuesta), y dichas personas consumen desde 10 hasta 40 pañales en ese intervalo de tiempo (pregunta 11 de la encuesta). El precio estimado de venta de la nueva almohadilla biodegradable propuesta en este estudio es de $\$ 3.95$ pesos por cada pieza, por tal motivo, el consumidor estaría gastando entre $\$ 40$ y $\$ 158$ pesos por semana.

\section{Conclusión}

Se demostró la capacidad de absorción del tallo maduro del Lirio Acuático, la cual es mejorada al adicionar un polisacárido con capacidad gelificante. 
En base a una encuesta aplicada a los consumidores potenciales de la nueva almohadilla absorbente desechable y biodegradable se concluye que este producto tendría una gran aceptación y un gran impacto social.

Se podría extender la aplicación del nuevo biomaterial absorbente desarrollado a toallas sanitarias biodegradables.

\section{References:}

1. Castro, J. (2016). Búsqueda preliminar de morfotipos fúngicos degradadores de sustratos celulósicos en residuos sólidos asociados a pañales desechables. Bogotá, Colombia.

2. Daulay, M., Rosmayanti, S., Ramayani , O. R., Supriatmo, S., Ramayati, R., \& Rusdidjas, R. (2013). Association between the frequency of disposable diaper changing and urinary tract infection in children. Paediatrica Indonesiana, 70-75.

3. Leiva-Mas, J., Martínez Nodal, P., Esperanza-Pérez, G., RodríguezRico, I.L., \& Gordiz-García, C. E. (2012). Absorción de hidrocarburos en columnas rellenas con bagazo: una solución sostenible. ICIDCA, $36-44$.

4. Márquez Rivera, C. A., \& Arguelles Robles, A. I. (2017). Evaluación del tallo maduro del lirio acuático (Eichhornia crassipes) como sorbente de hidrocarburos para el combate de derrames en la industria petrolera (tesis de maestría). Instituto de Estudios Superiores de Tamaulipas, Altamira, México.

5. Pozos Vázquez C. \& Bollnow Cortes A. H. (mayo, 2017). Biosorción del azul de metileno en solución acuosa mediante el uso del lirio acuático. Trabajo presentado en el $29^{\circ}$ Encuentro Nacional de Investigación Científica y Tecnológica del Golfo de México, Tampico, Tamaulipas. Resumen recuperado de file:///C:/Users/profesores.uac/Downloads/memoriaresumen\%2029\%20(3).pdf.

6. Ramos, C., García, R., Sandoval, G., Arellano, L., \& Antonio, R. (2014). Gel de carboximetilcelulosa (CMC) a partir del bagazo de piña. Ciencias de la Ingeniería y Tecnología, 202-208.

7. Rojas González, A. \& Osswald, T. (2015). Determinación de la degradación térmica de polímeros por análisis de cambio de color. Revista Ingeniería, 19-30.

8. Smith, M. V. (2013). Diaper need and its impact on child health. Pediatrics, 253-259. 